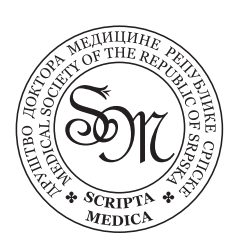

CASE REPORT

\title{
Surgical Treatment of Coronary Artery Aneurysm
}

\section{Živojin S. Jonjev',} Svetozar Srdić ${ }^{2}$

${ }^{1}$ University of Novi Sad, Institute for Cardiovascular Diseases of Vojvodina, Clinic of Cardiovascular Surgery, Sremska Kamenica, Serbia ${ }^{2}$ Clinical Center Banja Luka, Clinic for Cardiovascular Diseases, Banja Luka, Bosnia \& Herzegovina

\section{Correspondence}

Živojin S. Jonjev, MD, PhD

Clinic of Cardiovascular Surgery Institutski put 4

21204 Sremska Kamenica, Serbia

E-mail: jonjevz@nscable.net

Submitted: September 21, 2011

Accepted: September 26, 2011
A 59-years-old man was transferred from an outside hospital with recurrent postero-inferior myocardial infarction. On admission both physical exam and chest roentgenogram indicated ischemic cardiomyopathy (NYHA III/IV). An electrocardiogram confirmed normal sinus rhythm $(\mathrm{F}=86 / \mathrm{min})$ as well as an old myocardial infarction of the posterior wall of the left ventricle. Cardiac catheterization showed large coronary aneurysms on both the proximal right coronary artery (RCA) and the left anterior descending artery (LAD) along with RCA occlusion and significant LAD stenosis (>90\%), just below the aneurismal sac. The circumflex artery (RCx) had multiple stenoses, resulting in poor visualization of the distal RCx (Figure $1 \mathrm{a}$ and $\mathrm{b})$. Trans-thoracic echocardiography revealed decreased left ventricle function $(\mathrm{EF}=\mathbf{2 9 \%})$, and mild mitral and tricuspid regurgitation (I/II degree).

Despite the significantly increased perioperative mortality risk (EuroSCORE [E-log]=24.12\%), the patient underwent elective heart surgery. Upon exposure of the heart, two large, firm and partially calcified coronary aneurysms were found: one on the RCA $(\varnothing \approx 3 \mathrm{~cm})$ and one on the proximal $\operatorname{LAD}(\varnothing \approx 1.5 \mathrm{~cm})$. Myocardial revascularization was successfully achieved with double coronary bypass using the left internal mammary artery to graft the LAD site and a saphenous venous graft for the distal RCA site (Figure 2). The patient's postoperative course was uneventful. He was discharged from the hospital on postoperative day \#7 and remained stable three months after surgery.
Coronary artery aneurysm (CAA) is a very rare finding. It is usually described as a focal dilatation of the adjacent coronary artery that exceeds the diameter of the native coronary artery by 1.5 times or more. CAA could be highly symptomatic, or it might be diagnosed postmortem at autopsy. Registry studies indicate that the incidence of CAA is less than 5\%. However, improved imaging techniques suggest that the true incidence may be underestimated. Therapeutic modalities for CAA are still controversial and consist of medical therapy alone, coronary stenting or surgical reconstructive techniques. Our patient's disease was

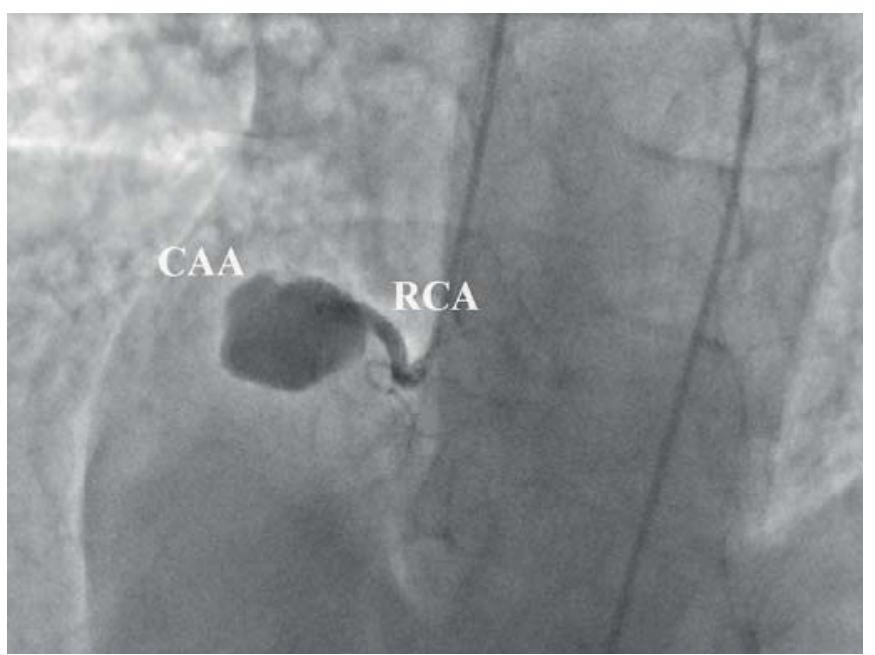

Figure 1a: Radiography of the RCA. Findings show occlusions of both the CAA and RCA just below the aneurysmal sac. RCA-right coronary artery; CAA-coronary artery aneurysm. 


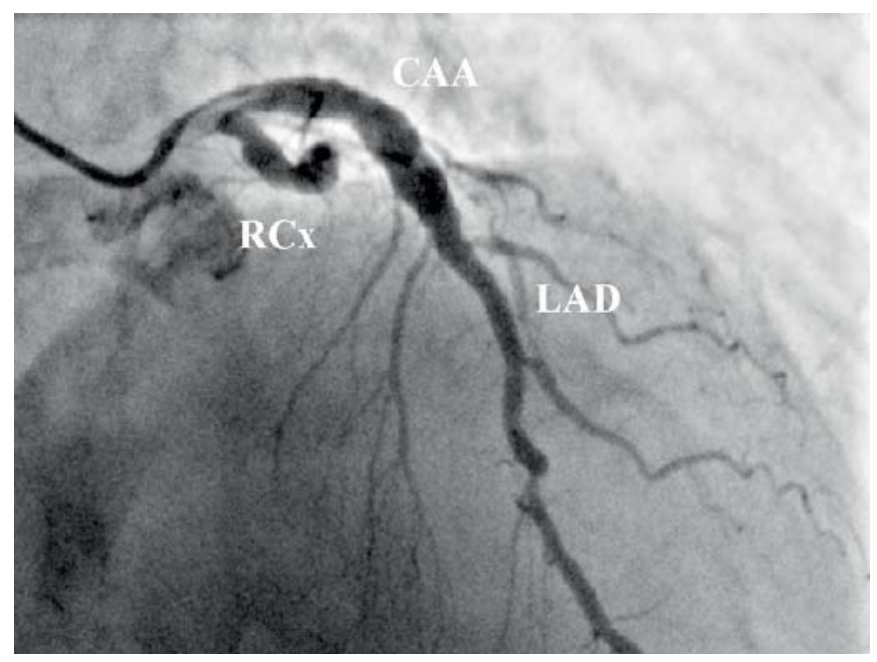

Figure 1b: Radiography of the left coronary artery. It shows poor visualization of the distal RCx, a large coronary artery aneurysm in the proximal LAD and significant coronary stenosis (>90\%) just below the aneurismal sac. LAD - left anterior descending artery; $\mathrm{RCx}$ - circumflex artery; CAA - coronary artery aneurysm.

caused by atherosclerosis; medical treatment alone in such individuals has only limited efficacy. Occlusion of the aneurysmatic nonstenotic coronary artery usually causes myocardial infarction, ${ }^{1}$ as it did in this particular case. Accordingly, surgical intervention should occur as soon as possible to reduce the risk of thromboembolic events, even in a setting of antiplatelet therapy.

Several surgical techniques have been described. ${ }^{2}$ Our choice is short and simple CABG surgery. This approach improves antegrade coronary flow distally to CAA, which prevents potential thromboemboli arising from the aneurismal sac and lowers the risk of myocardial ischemia. Good angiographic visualization, trans-esophageal echo-

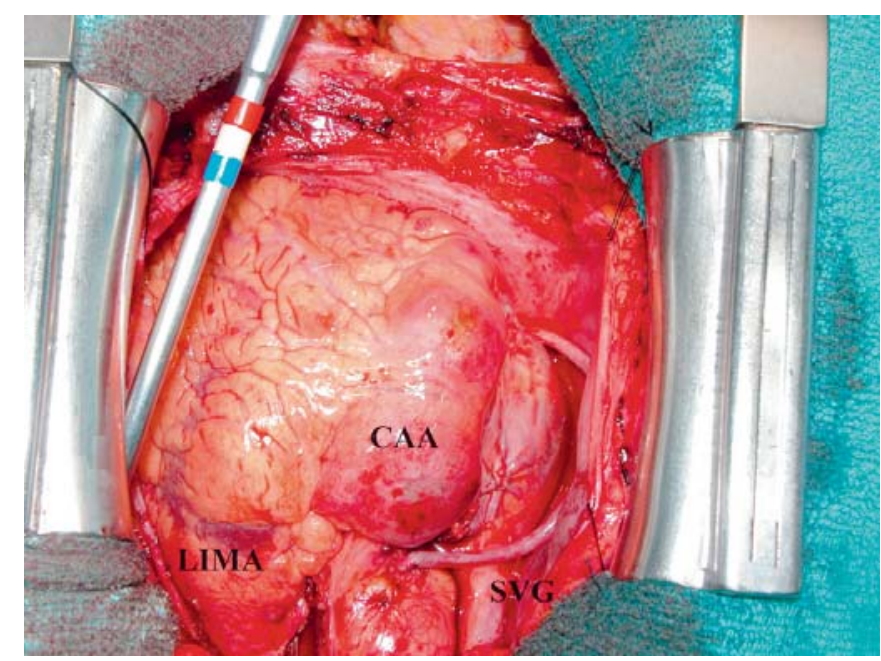

Figure 2: Intraoperative view. It shows a CAA of the right coronary artery repaired distally with a saphenous venous graft and the LAD repaired with a LIMA graft. CAA - coronary artery aneurysm; SVG - saphenous venous graft; LIMA - left internal mammary artery.

cardiography and magnetic resonance imaging can be used not only for diagnosis but for follow-up as well. We recommend surgery as soon as possible to prevent thromboembolic complications, especially in cases with severe coronary stenosis and persistent angina despite optimal medical therapy.

\section{References}

1. Brecker SJ, Gray HH, Oldershaw PJ. Coronary artery aneurysms and myocardial infarction: adult squeals of Kawasaki disease? Brit Heart J 1988; 59:509-12.

2. Ercan E, Tengiz I, Yakut $\mathrm{N}$ et al. Large atherosclerotic left main coronary aneurysm: a case report and review of literature. Int J Cardiol 2003; 88:95-8. 